\title{
IS THE NORMAL-TO-SUPERCONDUCTING TRANSITION OF FIRST OR SECOND ORDER?
}

\author{
R. Folk ${ }^{\dagger}$, Yu. Holovatch ${ }^{\dagger \dagger}$ \\ ${ }^{\dagger}$ Institut für Theoretische Physik, Johannes Kepler Universität Linz, A-4040 Linz, Austria \\ ${ }^{\dagger \dagger}$ Institute for Condensed Matter Physics of the Ukrainian Acad. Sci. \\ 1 Svientsitskii Str., Lviv UA-290011, Ukraine
}

(Received September 3, 1996)

\begin{abstract}
We study the phase transition to the superconducting state taking into account the fluctuations of the order parameter and of the vector magnetic field and discuss the question of the order of transition occuring in this model. We use the filed-theoretical renormalization group approach and consider the field-theoretical gauge model for a superconductor, generalized to a $n / 2$ component complex order parameter. Renormalization group calculations within strict $\varepsilon$-expansion suggested that in such a model a first-order phase transition occurs. We re-examine the previously obtained expressions for the renormalization group functions in a two-loop approximation in three dimensions. Special attention is being payed to the fact, that the corresponding series might be asymptotic ones and therefore have zero radius of convergence. We discuss possible ways of the analytical continuation of the series obtained. On the basis of the comparison of the results obtained by "direct" calculations with those obtained by Padé analysis and Padé-Borel resummation technique the conjecture is made that in the model under consideration still exists a possibility for the second-order phase transition with the critical exponents differing from those of a superfluid liquid. This is in agreement with conclusions made very recently in other nonperturbative treatments.
\end{abstract}

Key words: superconductivity, phase transitions, renormalization group.

PACS number(s): 05.70.Jk, 64.60.Fr, 74.20-z, 02.30.Mv

\section{INTRODUCTION}

The order of a phase transition may have severe consequences for physical quantities. So one knows from the first order liquid gas transition phenomena like overheating and undercooling connected with the metastability at the transition. For the second order phase transition divergencies in physical quantities occur (in the thermodynamic limit of course) leading to a dramatic increase of the specific heat or scattering of light (critical opalescence) near the liquid gas critical point.

Similar dramatic changes are connected with the phase transitions which occured in the early stage of the universe and the questions discussed here for the superconductor are also relevant there (for a recent review see [1]). However when the question raised in the title was discussed for the superconductor it was more or less an academic question, since due to the large correlation length $\left(\xi_{0} \sim 10^{3} \AA\right)$ only very near the phase transition the first order character can be seen, otherwise mean field beheavior is to be expected. This situation changed after the discovery of high- $\mathrm{T}_{c}$ superconductors with correlation lengths within the range of the lattice distances $\left(\xi_{0} \sim 1 \AA\right)[2]$. Here in several experiments critical effects have been observed in the specific heat $[3,4]$. They have been analysed [5] with scaling exponents related to the fixed point in the uncharged model of a superconductor which takes into account only the fluctuations of the complex order parameter and corresponds to the model describing the phase transition into the superfluid phase.

From the theoretical point of view according to BCS theory of superconductivity the normal-tosuperconducting (NS) phase transition is a classical second order phase transition described by the LandauGinsburg Hamiltonian with the complex order parameter, corresponding to the wave function of the Cooper pairs. Taking into account the fluctuations of the order parameter one can find the values of corresponding critical exponents which in this case will coincide with the critical exponents of $O(n)$ symmetrical field theoretical model for the case $n=2$. Consequentely this leads to the answer that NS phase transition is described by the same set of critical exponents as the phase transition in normal-to-superfluid liquid. The last are known with high accuracy [6] and calculated by different methods [7-9].

However taking into account, that for the NS transition the corresponding "superfluid liquid" is charged, essentially complicates the problem. For the first time this question was considered by B. I. Halperin, T. C. Lubensky and S. Ma [10] and since then different ways of tackling it were proposed. The theoretical model describing the relevant critical behaviour was the usual $O(n)$ symmetrical $\phi^{4}$ model with the $n / 2$-component complex field $\phi$ coupled to a gauge field describing the fluctuating magnetic field created by Cooper pairs. The answer obtained in [10] states that because of the coupling to the gauge field in mean field approximation a third order term ap- 
pears in the free energy of the superconductor and the NS phase transition is of the first order. This was coroborated by renormalization group theory from the results of a first order $\varepsilon$-expansion. A stable fixed point (neccessary, but not sufficient for a second order phase transition) exists only for the order parameter components number $n>365.9$, exceeding to a great extent the superconductor case $n=2$. The crossover near the first order phase transition was studied [11] and the expression for the crossover function of the specific heat was given within one loop order perturbation theory.

However the mean field results where questioned by a calculation of Lovesey [12], which showed that taking into account the gauge field fluctuations in the calculation of free energy leads back to a second order phase transition. A further indication of a second order phase transition came several years later when this problem was studied on the lattice by means of MC calculations and duality arguments [13]. The results confirmed scenarios of the NS transition differing from those obtained in [10]. Namely, the NS transition was found to be of the second order asymptotically equivalent to that of a superfluid with the reversed temperature axis. Subsequent MC simulations [14] performed in different regions of couplings lead to the result that the NS transition is strongly first order deep in the type-I region and becomes more weakly first order moving in the direction of the type-II region [15]. Beyond a certain point the data of [14] suggest a second-order transition. The corresponding $O(n)$ nonlinear $\sigma$-model coupled to an Abelian gauge field studied near two dimensions by $2+\varepsilon$ expansion [16] did not show a first order phase transition either.

The influence of the critical fluctuations on the order of NS transition was reconsidered on the basis of the ideas of field theoretical renormalization group in [17]. Here the two-loop flow equations [18] for the static parameters and the $\zeta$-functions [19] were obtained and it was indicated that a stable fixed point might be possible and, as a consequence, a second order phase transition might appear. An attractive feature of the flow found in [17] was that it discriminated between type-I and type-II superconductors, depending on the initial (background) values of the couplings. For small values of the ratio (coupling to the gauge field)/(fourth order coupling) (appropriate for typeII superconductors) the flow comes very near to the fixed point of the uncharged model but ends in the new superconducting fixed point. For large values of the ratio (type-I superconductors) the flow runs away. For values of the ratio in between the critical behaviour might be influenced by a second (unstable) supeconducting fixed point with scaling exponents quite different from the uncharged model. In the context of baryogenesis the problem of the order of the NS phase transition was considered in two loop order in [20] and the effective potential was calculated. Of course, the result was a first order phase transition since only run away flows are found in strict perturbation theory.

Coincedence of the critical exponents of the NS transition with those of a superfluid transition with reversed temperature axis was supported by the renormalization group arguments on the basis of dual fomulation of the Landau-Ginsburg theory [21]. Recently the same problem studied by the renormalization group technique in fixed dimension $d=3$ in one-loop approximation showed the evidence of an attractive charged fixed point distinct from that of a neutral superfluid leading, in particlar, to the correlation length critical exponent value $\nu \simeq 0.53$ and $\eta \simeq-0.70[22]$.

The problem of the NS transition was also studied [23] by means of the self-consistent screening approximation [24]. The approximation builds the $1 / n$ xpansion for the general dimensionality $d$. The anomalous field dimension exponent $\eta(d, n)$ was calculated and the result $\eta(3,2) \simeq-0.38$ was found. Near $d=4$ the results obtained were in good agreement with the $\varepsilon$-expansion data [10] for high $n(n>366)$ near $d=4$.

To conclude this brief review it is worth mentioning one more physical interpretation of a charged field coupled to a gauge vector potential. Namely it is the nematic-smectic A transition in liquid crystals [25]- [28]. It is believed that this transition is described by a model similar to those describing the NS transition in the charged case [10]: now the smectic order parameter is coupled to the director fluctuations. On contrary to the NS type transition, the nematic-smectic A transition is characterised by the critical region of the experimentally accesible range and appears to be continuous (see e.g. $[27,28])$. Further applications have been suggested in the context of the quantum Hall effect [29].

The purpose of our article is to show that even being within the frames of the renormalization group method applied to the original model of the superconductor minimally coupled to the gauge field [10] one still can obtain the answer that in such a model there occurs a second order phase transition with the critical exponents distinct from those of a superfluid liquid. To prove this we reconsider two-loop renormalization group functions obtained in [17] for this model [10] and pay special attention to the fact that the loop expansion is the asymptotic one [30]- [33]. We apply resummation technics to the betafunctions of the flow as well as to the zeta-functions. In this way we find several fixed points with new scaling exponents and a rich crossover behaviour. Some of our results were previously published in [34]. A similar approach, namely nonperturbative flow equations, where used in [35] and there also a stable fixed point was found.

The setup of the article is as follows. In the next section II we describe the model we are interested in, give the expressions for the renormalization group functions in a two loop approximation and describe the results obtained on their basis without applying any resummation procedure. In the section III we discuss the resummation technique. Section IV is devoted to a study of the $\beta$-functions which are the functions of two variables and of the corresponding flows on the basis of the Padé-Borel resummation technique and Padé approximants for the appropriate resolvent series. In section $\mathrm{V}$ we calculate the assymptotic and effective values for the critical exponents. The results are discussed in section VI. 


\section{THE MODEL AND ITS "NAIVE" ANALYSIS}

As is well known now the influence of the order parameter fluctuations on the NS transition can be described by the Landau-Ginsburg free energy functional:

$$
F[\phi]=\int \mathrm{d}^{3} x\left\{\frac{t_{0}}{2}\left|\phi_{0}\right|^{2}+\frac{1}{2} \mid\left(\left.\nabla \phi_{0}\right|^{2}+\frac{u_{0}}{4 !}\left|\phi_{0}\right|^{4}\right\},\right.
$$

$t_{0}$ being temperature-dependent, $u_{0}$ is a coupling constant and the complex order parameter $\phi_{0}$ is connected with the wave function of Cooper pairs. The Cooper pairs are charged and therefore create fluctuating magnetic field which leads to the appearence of additional terms in the free energy functional. Note that it is not the case for a normal-to-superfluid transition in neutral (uncharged) fluid, which is well described by (2.1) without any modifications. Describing the fluctuating magnetic field $\mathbf{B}$ by the vector potential $\mathbf{A}(\mathbf{B}=\operatorname{rot} \mathbf{A})$ and adding to (2.1) the minimal coupling between the fluctuating vector potential and the order parameter one gets the free energy functional $F[\Psi, \mathbf{A}]$ originally considered in [10] for a generalized superconductor in $d$ dimensions with the $d$-dimensional vector potential $\mathbf{A}$ and the order parameter $\Psi$ consisting of $n / 2$ complex components. Now one can describe the fluctuation effects by an Abelian Higgs model with the gauge invariant Hamiltonian [10]:

$$
\begin{aligned}
H & =\int \mathrm{d}^{d} x\left\{\frac{t_{0}}{2}\left|\Psi_{0}\right|^{2}+\frac{1}{2}\left|\left(\nabla-i e_{0} \mathbf{A}_{0}\right) \Psi_{0}\right|^{2}\right. \\
& \left.+\frac{u_{0}}{4 !}\left|\Psi_{0}\right|^{4}+\frac{1}{2}\left(\nabla \times \mathbf{A}_{0}\right)^{2}\right\},
\end{aligned}
$$

depending on the bare parameters $t_{0}, e_{0}, u_{0}$. The parameter $t_{0}$ changes its sign at some temperature, the rest of the parameters being considered as temperatureindependent. For the coupling constant $e_{0}=0$ no magnetic fluctuations are induced and the model reduces to the usual field theory (2.1) describing a second-order phase transition and corresponding the particular case $n=2$ to the superfluid transition in ${ }^{4} \mathrm{He}$.

In order to describe long-distance properties of model (2.2) arising in the vicinity of the phase transition point we apply field-theoretical renormalization group approach. Recent two-loop results [17] for the renormalization group functions corresponding to $(2.2)$ were obtained on the basis of dimensional regularization and minimal subtraction scheme [36] (for calculations at $d=$ 3 see $[37,38]$ ) introducing renormalized fields and couplings by:

$$
\begin{aligned}
& \Psi_{0}=Z_{\Psi}^{1 / 2} \Psi, \quad \mathbf{A}_{0}=Z_{\mathrm{A}}^{1 / 2} \mathbf{A} \\
& t_{0}-t_{0 c}=Z_{t} Z_{\Psi}^{-1} t \\
& e_{0}^{2}=Z_{e}^{1} Z_{\mathrm{A}}^{-1} Z_{\Psi}^{-1} e^{2} \mu^{\varepsilon} S_{d}^{-1} \\
& u_{0}=Z_{u} Z_{\Psi}^{-2} u \mu^{\varepsilon} S_{d}^{-1} .
\end{aligned}
$$

with $\varepsilon=4-d$. Here $\mu$ is a reference wave number, $t_{0 c}$ being a shift, which for the results considered here can be set to zero, and $S_{d}$ stands for the surface of $d$-dimensional hypersphere: $S_{d}=2^{1-d} \pi^{-d / 2} / \Gamma(d / 2)$. The $Z$-factors are determined by the condition that all poles at $\varepsilon=0$ are removed from the renormalized vertex functions. From a Ward identity one has $Z_{\Psi}=Z_{e}$, and the remaining $Z$-factors are to be found from the corresponding vertex functions. The results in two-loop order found in [17] read:

$$
\begin{aligned}
Z_{\Psi} & =1+\frac{1}{\varepsilon}\left\{3 e^{2}-u^{2}(n+2) / 144+e^{4}[(n+18) / 4 \varepsilon-(11 n+18) / 48]\right\}, \\
Z_{\mathrm{A}} & =1+\frac{1}{\varepsilon}\left\{-n e^{2} / 6-n e^{4} / 2\right\}, \\
Z_{t} & =1+\frac{1}{\varepsilon}\left\{(n+2) u / 6+u^{2}[(n+2)(n+5) / 36 \varepsilon-(n+2) / 24]\right. \\
& \left.+u e^{2}[-(n+2)(1 / 2 \varepsilon-1 / 3)]+e^{4}[(3 n+6) / 2 \varepsilon+(5 n+1) / 4]\right\}, \\
Z_{u} & =1+\frac{1}{\varepsilon}\left\{(n+8) u / 6+18 e^{4} / u+u^{2}\left[(n+8)^{2} / 36 \varepsilon-(5 n+22) / 36\right]\right. \\
& +u e^{2}[-(n+8) / 2 \varepsilon+(n+5) / 3]+e^{4}[(3 n+24) / \varepsilon+(5 n+13) / 2] \\
& \left.+e^{6} / u[3(n+18) / \varepsilon-7 n / 2-45]\right\} .
\end{aligned}
$$


The flow equations for the renormalized couplings $u$, $f\left(f=e^{2}\right)$ are:

$$
\begin{aligned}
& l \frac{d f}{d l}=\beta_{f}, \\
& l \frac{d u}{d l}=\beta_{u},
\end{aligned}
$$

where $l$ is the flow parameter and the expressions for $\beta$-functions in two-loop approximation read [17]:

$$
\begin{aligned}
& \beta_{f}=-\varepsilon f+\frac{n}{6} f^{2}+n f^{3}, \\
& \beta_{u}=-\varepsilon u+\frac{n+8}{6} u^{2}-\frac{3 n+14}{12} u^{3}-6 u f+18 f^{2} \\
& +\frac{2 n+10}{3} u^{2} f+\frac{71 n+174}{12} u f^{2}-(7 n+90) f^{3} .
\end{aligned}
$$

The previous analysis of the equations of type (2.10), (2.11) either on one-loop [10] or on two-loop level [17] was based on direct solutions of the equation for the fixed point. In the present study we want to attract attention to the fact that the series have zero radius of convergence and they are known to be asymptotic at best. Therefore some additional mathematical methods have to be applied in order to obtain reliable information on their basis.

We start by recalling the results of a $\varepsilon^{2}$-expansion for $\beta$-functions $[10,17]$. In second order in $\varepsilon$ one obtains three fixed points: the Gaussian $\left(u^{* G}=f^{* G}=0\right)$, the "Uncharged" $\left(u^{* U} \neq 0, f^{* U}=0\right)$ and the "Charged" $\left(u^{* C} \neq 0, f^{* C} \neq 0\right)$, to be denoted as G, U, C. The expressions for them read:

$$
\begin{aligned}
& \mathrm{G}: \quad u^{* G}=0, \quad f^{* G}=0, \\
& \mathrm{U}: u^{* U}=u_{1}^{U} \varepsilon+u_{2}^{U} \varepsilon^{2}, f^{* U}=0, \\
& \mathrm{C}: u^{* C}=u_{1}^{C} \varepsilon+u_{2}^{C} \varepsilon^{2}, f^{* C}=f_{1}^{C} \varepsilon+f_{2}^{C} \varepsilon^{2},
\end{aligned}
$$

where

$$
\begin{aligned}
& u_{1}^{U}=\frac{6}{n+8}, \quad u_{2}^{U}=\frac{18(3 n+14)}{(n+8)^{3}}, \\
& u_{1}^{C}=\frac{3(n+36)+\left(n^{2}-360 n-2160\right)^{1 / 2}}{3 n(n+8)}, \\
& u_{2}^{C}=\frac{a_{2}}{a_{1}}, \quad f_{1}^{C}=\frac{6}{n}, \quad f_{2}^{C}=-\left(\frac{6}{n}\right)^{3} n,
\end{aligned}
$$

$$
\begin{aligned}
& a_{1}=1+\frac{n+8}{3} u_{1}^{C}-\frac{36}{n} \\
& a_{2}=\frac{3 n+14}{12}\left(u_{1}^{C}\right)^{3}-6 n u_{1}^{C}\left(\frac{6}{n}\right)^{3}+36 n\left(\frac{6}{n}\right)^{4}-\frac{(n+5) 4}{n}\left(u_{1}^{C}\right)^{3}-\frac{3(71 n+174)}{n^{2}} u_{1}^{C}+\left(\frac{6}{n}\right)^{3}(7 n+90) .
\end{aligned}
$$

Almost all physical results concerning phase transition described by the field theory $(2.2)$ were to some extend based on the information given by (2.12)-(2.14). The main of them read:

(i) fixed point $\mathrm{U}$ is unstable with respect to the presence of $f$-symmetry at $d<4$ with the stability exponent

$$
\lambda_{f}\left(u=u^{* U}, f=f^{* U}=0\right)=\left.\frac{\partial \beta_{f}}{\partial f}\right|_{U}=-\varepsilon
$$

(ii) fixed point $\mathrm{C}$ appears to be complex for $n<n_{c}=$ 365.9 [10] already on one-loop level. The stability exponent given by

$$
\lambda_{u}\left(u=u^{* C}, f=f^{* C}\right)=\left.\frac{\partial \beta_{u}}{\partial u}\right|_{C}
$$

and on the two-loop level it reads:

$$
\lambda_{u}=-\varepsilon s, \quad s=\left[\left(1+\frac{36}{n}\right)^{2}-\frac{432(n+8)}{n^{2}}\right]^{1 / 2}
$$

leading to an oscillatory flow in $u$ in one-loop order below $n_{c}$ with the solution $[11,17]$ : 


$$
\begin{aligned}
& f(l)=\frac{6 f l^{-\varepsilon}}{6+n \varepsilon f\left(l^{-\varepsilon}-1\right)}, \\
& u(l)=f(l) \frac{n}{2(n+8)}\left\{s \operatorname { t a n } \left[\frac{s}{2} \ln \left(f(l) f^{-1} l^{\varepsilon}\right)\right.\right. \\
& \left.+\arctan \left(\frac{2(n+8)}{s n} \frac{u}{f}+\frac{n+36}{n s}\right)\right] \\
& \left.-\frac{n+36}{n}\right\}
\end{aligned}
$$

here $f$ and $u$ are the initial parameters at $l=1$;

(iii) from the condition of positiveness of the fixed point coordinate $f^{*}\left(f=e^{2}\right)$ follows that at $\varepsilon=1$ $n>36$.

Finally the conclusion follows that for the "superconductor" case $n=2$ being of most physical interest there does not exist a stable fixed point and therefore the observed phase transition is of the first order.

\section{RESUMMATION}

Nevertheless one should note that such a staightforward interpretation of the $\varepsilon$-expansion data was questioned and a way of analyzing the series for $\beta$-functions (2.10),(2.11) avoiding strict $\varepsilon$-expansion and exploiting the information on the accurate solution for the pure model case at $d=3$ was proposed [17]. Also from the comparison of $\varepsilon$-expansion data for $f^{*}$ (giving positive value of $f^{*}$ only for $n>36$ ) with the value of $f^{*}$ obtained without $\varepsilon$-expansion (remaining positive for all $n$ ) the conjecture was made that the lower boundary for $n$ resulting in the negative $f^{*}$ might be an artifact of the expansion procedure.

As is well known now the appropriate resummation technique applied in the theory of critical phenomena to the asymptotic series for the renormalization group functions enables one to obtain extremly accurate values of the critical exponents. In fact the asymptotic nature of the series for the renormalization group functions has been proved only in the case of the $\phi^{4}$ model containing one coupling of $O(n)$-symmetry ( $n$-vector model) as well as the high-order asymptotics for these series is known $[32,31,33]$ in analytical form. These results gave the possibility to obtain precise values of the critical exponents for the $n$-vector model by the resummation of the corresponding series for the renormalization group functions (see e.g. $[7,8,40]$ ). For the "charged" model we are considering here up to our knowledge no information similar to those obtained in $[32,31,33]$ for the "uncharged" case $(f=0)$ is available. Nevertheless in the case of the model containing several couplings of different symmetry the asymptotic nature of the corresponding series for the renormalization group functions is rather a general belief than a proven fact. As one of such examples we mention here the weakly diluted $n$-vector model, described by a Hamiltonian containing two fourth order terms of different symmetry [39]. The asymptotic nature of the double series for the renormalization group functions in terms of the coupling constants has not been proven for this model up till now [41]. Nevertheless the appropriate resummation technique (applied as if these series are the asymptotic ones) enables one to obtain accurate values for critical exponents in three dimensions [42]- [45] and to describe (in $n=1$ case) the experimentally observed crossover to a new type of critical behaviour caused by weak dilution $[46,47]$. These results are also confirmed by Monte-Carlo $[48,49]$ and Monte-Carlo renormalization group [50] calculations.

Two main ways of resummation commonly used in these problems are: (i) resummation based on the conformal mapping technique and (ii) Padé-Borel resummation. The case (i) is based on the conformal transformation, which maps a part of the domain of analyticity containing the real positive axis onto a circle centered at the origin and the asymptotic expansion for a certain function is thus re-writen in the form of a new series (see [8] for discussion). However this resummation is based on the knowledge of subtle details of asymptotics (location of the pole, high-order behaviour) which are not avaible in our case.

In the absence of any knowledge about the singularities of the series the most appropriate method which can be used to perform the analytical continuation is the Padé approximation resulting in Padé-Borel resummation technique (ii) (see e.g. [7]). In the following we are going to apply it for the special case of $f=0$ so let us concentrate on it in detail.

Starting from the Taylor series for the function $f(u)$ :

$$
f(u)=\sum_{j \geq 0} c_{j} u^{j}
$$

one constructs the Borel transform

$$
F(u t)=\sum_{j \geq 0} \frac{c_{j}}{j !}(u t)^{j}
$$

Then one represents (3.2) in the form of Padé approximant $F_{[L / M]}^{P \text { Padé }}(u t)$ :

$$
F_{[L / M]}^{P a d e ́}(x)=\frac{\sum_{i=0}^{L} a_{i} x^{i}}{\sum_{j=0}^{M} b_{j} x^{j}}
$$

(in the subsequent analysis, proceeding in two-loop approximation we will use the [1/1] Padé approximant) and the resummed function is given by:

$$
f^{\text {Res }}(u)=\int_{0}^{\infty} d t e^{-t} F^{\text {Padé }}(u t) .
$$




\section{FIXED POINTS AND FLOWS IN THREE DIMENSIONS}

We will proceed here by considering the flow equations (2.8),(2.9) direcly at $d=3$. Let us look for the solutions of the fixed point equations at $d=3$ paying attention to the possible asymptotic nature of the corresponding series $(2.10),(2.11)$. Consider first the equation for the uncharged fixed point $U$. Substituting value $f^{*}=0$ into (2.11) one obtains the following expression for the function $\beta_{u}^{U} \equiv \beta_{u}\left(u, f^{*}=0\right)$ :

$$
\beta_{u}^{U}=-u+\frac{n+8}{6} u^{2}-\frac{3 n+14}{12} u^{3}
$$

Solving this polynomial for the fixed point one obtains for the non-trivial $u^{*}>0$ :

$$
u^{* U}=\frac{n+8}{3 n+14}+\frac{\sqrt{n^{2}-20 n-104}}{3 n+14}
$$

and immidiately the "condition of the existence of nontrivial solution $u^{* U "}$ qualitatively very similar to those, appearing in the frames of the $\varepsilon$-expansion technique (see $[10,17]$ and formula (2.13) of the present article as well) follows : the solution exists only for certain values of $n>n_{c}=24.3 \quad$ ! From fig. 1 one can see that the function $\beta_{u}^{U}$ (4.1) does not intersect the $u$-axis at any non-zero value of $u$ for $n=2$. In the $O(n)$-symmetric $\phi^{4}$-theory at $d=3$ this situation is well-known (see e.g. [51,52]): the $\beta$-function calculated directly at $d=3$ does not possess a stable zero for the realistic values of $n$, nevertheless in three-loop order the presence of the stable fixed point is restored. To avoid this artifact appearing in the twoloop calculation one can either resume the series for $\beta$ function or construct the appropriate Padé approximant [53] in order to perform the analytical continuation of (4.1) out of the domain of convergence (which is equal to zero for the series in the right-hand side of (4.1). Let us try both ways. Representing (4.1) in the form of $[1 / 1]$ Padé approximant:

$$
\beta_{u}^{U, \text { Padé }}=u \frac{-1+A_{u} u}{1+B_{u} u}
$$

one obtains:

$$
A_{u}=\frac{n^{2}+7 n+22}{6(n+8)}, \quad B_{u}=\frac{3 n+14}{2(n+8)}
$$

and, solving the equation for the fixed point:

$$
\beta_{u}^{U, \text { Padé }}\left(u^{* \text { P,Padé }}\right)=0
$$

one obtains:

$$
u^{* U, \text { Padé }}=\frac{6(n+8)}{n^{2}+7 n+22}
$$

So we obtained a qualitatively different situation. The behaviour of the function $\beta_{u}^{U, P a d e ́}(u)$ for $n=2$ is shown in fig. 1 by the dashed curve. If one is interested in more accurate values of $u^{*}$ some resummation has to be applied. Choosing the Padé-Borel resummation technique [54] and following schemes (3.1)-(3.4) one obtains for the resummed function $\beta_{u}^{U, \text { Res }}$ :

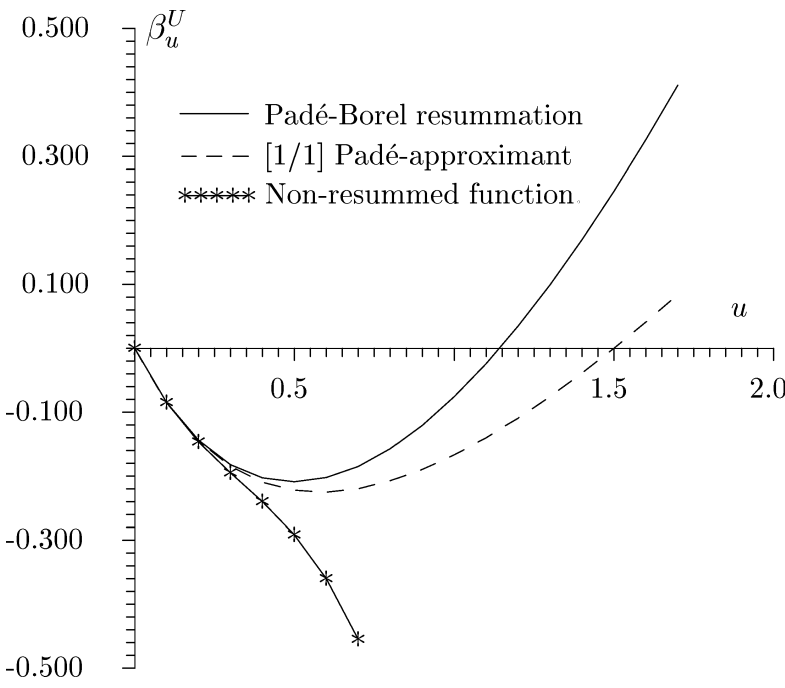

Fig. 1. $\beta_{u}$-function of the uncharged model $\beta_{u}^{U}$ at $d=3$, $n=2$.

$$
\beta_{u}^{U, \text { Res }}=u\left[2\left(1-A_{u} / B_{u}\right)\left(1-E\left(\frac{2}{u B_{u}}\right)\right)-1\right]
$$

the coefficients $A_{u}, B_{u}$ are given by (4.4), $E(x)=$ $x e^{x} E_{1}(x)$, where the function

$$
E_{1}(x)=e^{-x} \int_{0}^{\infty} d t e^{-t}(x+t)^{-1}
$$

is connected with the exponential integral by the relation [55]:

$$
E_{1}(x \pm i 0)=-E i(-x) \mp i \pi
$$

The behaviour of the function $\beta_{u}^{U, \text { Res }}(u)$ is shown in fig. 1 by the solid curve. And the fixed point coordinate $u^{* U, \text { Res }}$ is obtained solving the non-linear equation:

$$
\beta_{u}^{U, \operatorname{Res}}\left(u^{* U, \operatorname{Res}}\right)=0 .
$$

The coordinates of the fixed point $u^{* U}$ obtained on the basis of Padé approximation and Padé-Borel resummation $\left(u^{* U, P a d e ́}, \quad u^{* U, R e s}\right)$ for different $n$ are given in Table I.

We conclude from this analysis: in $d=3$ theory Padé approximants (as an analytical continuation of $\beta$ functions) qualitatively may change the picture and lead to the values of fixed points comparable to those obtained by the Padé-Borel resummation technique. 
Consider now the equation for the charged fixed point $C$ applying the above considerations to $\beta_{f}$ for which the expression at $d=3$ reads $(2.10)$ :

$$
\beta_{f}=-f+\frac{n}{6} f^{2}+n f^{3} .
$$

The behaviour of $\beta_{f}$ as a function of $f$ is shown in fig. 2 by asterisks. Note however that in this case the function $\beta_{f}$ even without any resummation possess a non-trivial zero $f^{* M}$ (its value $f^{* C, D i r}$ is given in the 2 nd column of Table II). Representing (4.9) in the form of [1/1] Padé approximant:

$$
\beta_{f}^{\text {Padé }}=f \frac{-1+A_{f} f}{1+B_{f} f}
$$

one has for $A_{f}, B_{f}$ :

$$
A_{f}=\frac{n+36}{6}, \quad B_{f}=-6,
$$

and, solving the equation for the fixed point coordinate $f^{* C, \text { Padé }}$ :

$$
\beta_{f}^{\text {Padé }}\left(f^{* C, \text { Padé }}\right)=0
$$

one obtains:

$$
f^{* C, \text { Padé }}=\frac{6}{n+36} .
$$

The function $\beta_{f}^{\text {Pade }}(f)$ is shown in fig. 2 by the dashed line, the coordinate $f^{* C \text {,Padé }}$ is given in the 3 rd column of Table II. But now the series (4.9) is not alternating and this results in the presence of a pole (at $f=\frac{1}{6}$ ) in the approximant (4.10). Therefore (4.10) correctly represents the function $\beta_{f}(f)$ only for $f<1 / 6$. Let us note however that for all the positive $n$ a fixed point exists and its coordinate $f^{* M, \text { Padé }}$ lies within the limits $0<f^{* C, \text { Padé }}<1 / 6$, where no pole in (4.10) exists. Comparing this result with those obtained for the uncharged fixed point one can note that the representation of $\beta_{f}$ in the form of the Padé approximant does not qualitatively change the picture (a solution for $\beta_{f}(f)=0$ exists at $d=3$ even without an analytical continuation) but results in a decrease of the fixed point coordinate. Contrary to the $\varepsilon$-expansion values (2.14) there does not exist any border line values of $n$ for the positivity of $f^{* C}$. Unfortunately we can not check this result by means of Padé-Borel resummation technique: the above mentioned presence of a pole in the denominator of the Padé approximant makes the corresponding integral representation problematic [56]. In order to find the $u$-coordinate of the fixed point $\mathrm{C}, u^{* C}$, we have to deal with a function of two variables, $\beta_{u}(u, f)$, represented by a rather short series (2.11). Another problem arises due to the fact that function $\beta_{u}(u, f)$ contains generating terms (i.e. $\left.\beta_{u}(u=0, f) \neq 0\right)$. In order to perform some kind of the analytic continuation of the function of two variables one can use rational approximants of two variables the (socalled Canterbury approximants or generalized Chisholm approximants $[57,58]$ ) which are generalization of Padé approximants in the case of several variables. But the presence of generating terms makes this choise rather ambiguous. The most reliable way in such a case seems to be the representation of $\beta_{u}(u, f)$ in the form of a "resolvent" series $B(u, f, t)[58,59]$ introducing an auxiliary variable $t$, which allows to separate contributions from different orders of the perturbation theory in the coupling constant. The series for $B(u, f, t)$ then reads:

$$
B(u, f, t) \equiv \beta_{u}(u t, f t)=\sum_{j \geq 0} b_{j} t^{j}
$$

with obvious notations for the coefficients $b_{j}$. Now one considers (4.14) as a series in the single variable $t$. This series can be represented in the form of Padé approximant $B^{\text {Padé }}(u, f, t)$ as the analytical continuation of the function $B(u, f, t)$ for the general value of $t$. In particular at $t=1$ the equality holds $B(u, f, t=1)=\beta_{u}(u, f)$ and the approximant

$$
B^{\text {Padé }}(u, f, t=1) \equiv \beta_{u}^{\text {Padé }}(u, f)
$$

represents the initial function $\beta_{u}(u, f)$. In our case the expression for $B(u, f, t)$ reads:

$$
B(u, f, t)=t\left(b_{1}+b_{2} t+b_{3} t^{2}\right)
$$

where:

$$
\begin{gathered}
b_{1}=-u, \quad b_{2}=\frac{n+8}{6} u^{2}-6 u f+18 f^{2}, \\
b_{3}=-\frac{3 n+14}{12} u^{3}+\frac{2 n+10}{3} u^{2} f \\
+\frac{71 n+174}{12} u f^{2}-(7 n+90) f^{3} .
\end{gathered}
$$

Representing the expression in brackets in the right-hand side of (4.15) in form of a [1/1] Padé approximant we have:

$$
B^{\text {Padé }}(u, f, t)=t b_{1} \frac{1+A_{u, f} t}{1+B_{u, f} t},
$$

where

$$
A_{u, f}=\frac{b_{2}}{b_{1}}-\frac{b_{3}}{b_{2}}, \quad B_{u, f}=\frac{-b_{3}}{b_{2}} .
$$




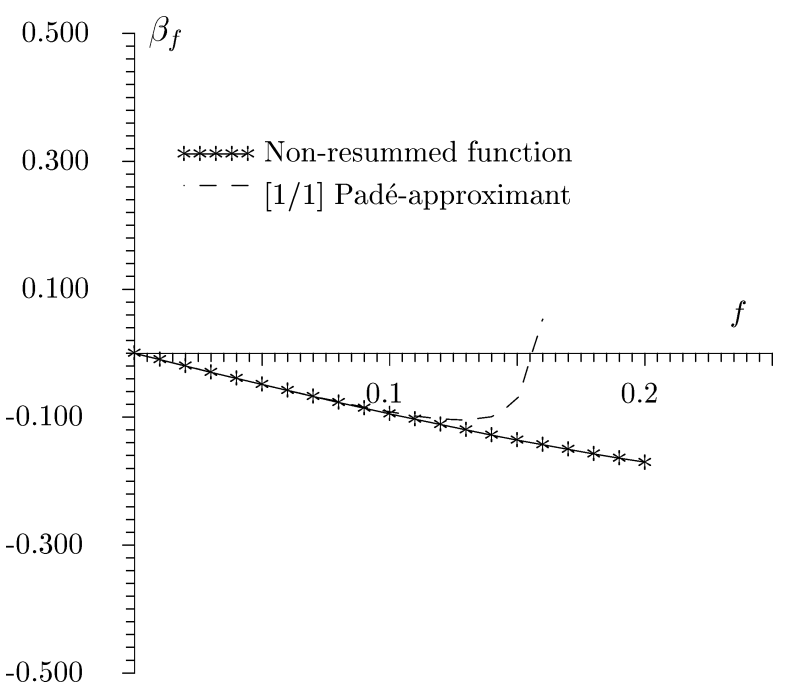

Fig. 2. $\beta_{f}$-function at $d=3, n=2$.

\begin{tabular}{lrr}
\hline \hline$n$ & $u^{* U, \text { Padé }}$ & $u^{* U, \text { Res }}$ \\
\hline 1 & 1.800 & 1.315 \\
2 & 1.500 & 1.142 \\
3 & 1.269 & 1.002 \\
4 & 1.091 & .888 \\
5 & .951 & .794 \\
6 & .840 & .717 \\
7 & .750 & .652 \\
8 & .676 & .597 \\
\hline \hline
\end{tabular}

Table I. Fixed point $\mathrm{U}$ coordinate $u^{* U}$ as a function of $n$. $u^{* U, \text { Padé }}:$ obtained on the basis of [1/1] Padé approximant; $u^{* U, R e s}$ : obtained by Padé-Borel resummation.

\begin{tabular}{rrrrr}
\hline \hline$n$ & $f^{* \text { C,Dir }}$ & $f^{* C, \text { Padé }}$ & $f^{* C, \varepsilon}$ & $f^{* C, \varepsilon^{2}}$ \\
\hline 1 & .920 & .162 & 6.000 & -210.000 \\
2 & .629 & .158 & 3.000 & -51.000 \\
3 & .500 & .154 & 2.000 & -22.000 \\
4 & .424 & .150 & 1.500 & -12.000 \\
5 & .372 & .146 & 1.200 & -7.440 \\
6 & .333 & .143 & 1.000 & -5.000 \\
7 & .304 & .140 & .857 & -3.551 \\
8 & .280 & .136 & .750 & -2.625 \\
\hline \hline
\end{tabular}

Table II. Fixed point C coordinate $f^{* C}$ as a function of $n$. $f^{* C, D i r}:$ obtained by direct solution of the equation for fixed point; $f^{* C, \text { Padé}}$ : obtained on the basis of $[1 / 1]$ — Padé approximant; $f^{* C, \varepsilon}: \varepsilon$-expansion result with the linear accuracy in $\varepsilon ; f^{* C, \varepsilon^{2}}: \varepsilon$-expansion result with the square accuracy in $\varepsilon$.

Let us note here that the function $B(u, f, t)$ obtained in this way as the approximant for the function of two variables $\beta_{u}(u, f)$ obeys certain projection properties in the single-variable case: substituting $f=0$ or $u=0$ into (4.16) one obtains the [1/1] Padé approximant for $\beta_{u}^{U}(u)$ or the $[0 / 1]$ Padé approximant for $\beta_{u}(u=0, f)$. Finally the expression for $\beta_{u}(u, f)$ approximated in such a way reads:

$$
\beta_{u}^{\text {Padé }}(u, f)=b_{1} \frac{1+A_{u, f}}{1+B_{u, f}} .
$$

Substituting into the equation for the fixed point $\beta_{u}\left(u^{* C}, f^{* C}\right)=0$ the value for the coordinate $f^{* C}=$ $f^{* C, \text { Padé }}$ (4.13) one obtains the non-linear equation for $u^{* C, \text { Padé }}$

$$
\beta_{u}^{\text {Padé }}\left(u, f=f^{* C, \text { Padé }}\right)=0 .
$$

Solving (4.19) with respect to $u$ one obtains the values $u^{* C, P a d e ́}$ given in Table III. The intersection of the function $\beta_{u}^{\text {Padé }}(u, f)(4.18)$ with the plane $f=f^{* C \text {,Padé }}$ is shown for $n=2$ in fig. 3 . The first fixed point (C1) given in the 2nd column of Table III turns out to be unstable, while the fixed point $\mathrm{C} 2$ is stable also for the case $n=2$ we are mainly interested in.

The crossover to the asymptotic critical behaviour is described by the solutions of the flow equations (2.8), (2.9) with the initial values of $u\left(\ell_{0}\right)$ and $f\left(\ell_{0}\right)$ at $\ell=\ell_{0}$ [60]. Substituting for the $\beta$-functions entering the righthand side of (2.8), (2.9) their analytical continuation in form of the Padé approximants (4.10), (4.18) we get the following system of differential equations:

$$
\begin{aligned}
& l \frac{d f}{d l}=f \frac{-1+A_{f} f}{1+B_{f} f} \\
& l \frac{d u}{d l}=-u \frac{1+A_{u, f}}{1+B_{u, f}}
\end{aligned}
$$

where $A_{f}, B_{f}$ and $A_{u, f}, B_{u, f}$ are given by (4.11) and (4.17) correspondingly.

Solving equations (4.20), (4.21) numerically one gets the flow diagram shown in fig. 4 for the case of $n=2$. The space of couplings is divided into several parts by separatrices (thick lines in fig. 4) connecting the fixed points. Besides the Gaussian $(\mathrm{G})$ there exist three fixed points, one corresponding to the uncharged (U) and two other corresponding to the charged $(\mathrm{C} 1, \mathrm{C} 2)$ cases. The fixed points $\mathrm{G}, \mathrm{C} 1$ and $\mathrm{U}$ are unstable (solid circles in fig. 4) and the fixed point $\mathrm{C} 2$ is the stable one (shown as a solid box in fig. 4). Several different flow lines are shown in fig. 4. They can be compared with the corresponding flow picture obtained by a direct solution of the flow equations for the two-loop $\beta$-functions expressed by the third-order polynomials in couplings $u, f(2.10)$, (2.11) (see fig. $2 \mathrm{a}$ in [17]). There one can see that no stable fixed point existed and even the fixed point $U$ was absent. Comparing fig. 4 and fig. $2 \mathrm{~b}$ from [17] one can see how an analytical continuation of the $\beta$-functions (2.9), (2.10), done only partly in [17] and performed here in the form of Padé approximants restores the presence of the fixed point $\mathrm{U}$ (unstable) and leads to the apperance 
of a new stable fixed point $\mathrm{C} 2$ for the charged model. The coordinates of the fixed points $\mathrm{U}, \mathrm{C} 1, \mathrm{C} 2$ are given in the corresponding columns of tables I, II, III and for $n=2$ they are equal to:

\begin{tabular}{lrr}
\hline \hline$n$ & \multicolumn{3}{c}{$u^{* C, \text { Padé }}$} \\
\hline 1 & $\mathrm{C} 1$ & $\mathrm{C} 2$ \\
2 & .184 & 3.309 \\
3 & .181 & 2.457 \\
4 & .179 & 1.781 \\
5 & .177 & 1.150 \\
6 & .175 & 0.473 \\
7 & .175 & 0.369 \\
8 & .176 & 0.305 \\
\hline \hline
\end{tabular}

Table III. Fixed point C coordinates $u^{* C \text {, Padé }}$ obtained on the basis of [1/1] Padé approximant for the "resolvent" series as a function of $n$. C1 : unstable fixed point; C2 : stable fixed point.

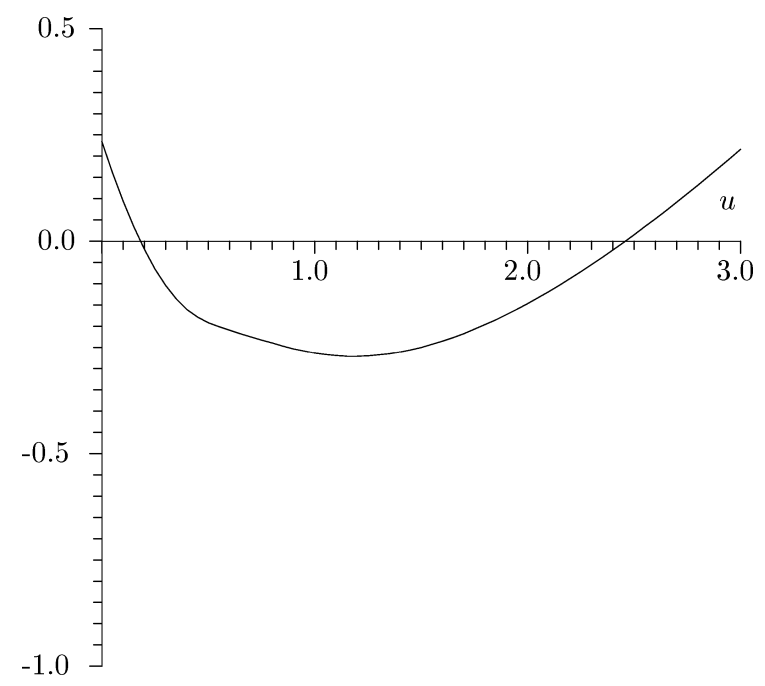

Fig. 3. Intersection of the function $\beta_{u}^{\text {Padé }}(u, f)$ at $d=3$, $n=2$ with the plain $f=f^{* C \text {, Padé }}$ in two-loop approximation.

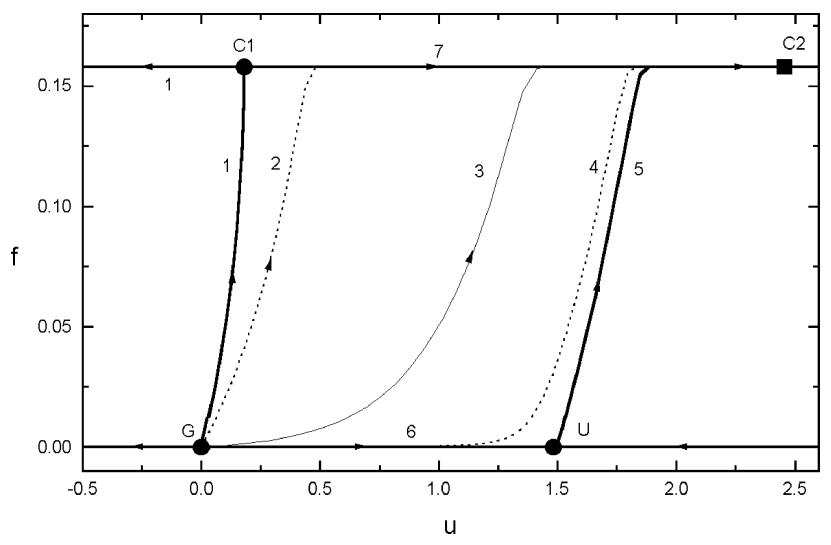

Fig. 4. Flow lines for the case $n=2, d=3$ given by equations (4.20), (4.21) (for further description see the text).

$$
\begin{aligned}
& \mathrm{U}: u^{*}=1.500, f^{*}=0, \\
& \mathrm{C} 1: u^{*}=.181, f^{*}=.158, \\
& \mathrm{C} 2: u^{*}=2.457, f^{*}=.158 .
\end{aligned}
$$

\section{CRITICAL EXPONENTS}

The values of critical exponents can be determined by the fixed point values of the $\zeta$-functions defined on the basis of renormalizing $Z$-factors (2.4) - (2.8) by:

$$
\zeta_{i}=\mu \partial \ln Z_{i} / \partial \mu
$$

where the derivative is taken at fixed unrenormalized couplings. The expressions for the $\zeta$-functions related to the order parameter and the temperature field renormalization in two-loop approximation read [17]:

$$
\begin{aligned}
\zeta_{\psi}= & -3 f+\frac{(n+2)}{72} u^{2}+\frac{(11 n+18)}{24} f^{2}, \\
\zeta_{t}= & \frac{-(n+2)}{6} u+\frac{(n+2)}{12} u^{2}- \\
& \frac{2(n+2)}{3} u f-\frac{(5 n+1)}{2} f^{2}, \\
\zeta_{A}= & \frac{n}{6} f+n f^{2} .
\end{aligned}
$$

If there exists a stable fixed point, the critical exponent $\nu$ of the correlation length, the critical exponent $\gamma$ of the order parameter susceptibility and the critical exponent $\alpha$ of the specific heat are given by:

$$
\begin{aligned}
& \nu=\left(2-\zeta_{\nu}^{*}\right)^{-1}, \\
& \gamma=\left(2-\zeta_{\nu}^{*}\right)^{-1}\left(2-\zeta_{\psi}^{*}\right), \\
& \alpha=\left(2-\zeta_{\nu}^{*}\right)^{-1}\left(\varepsilon-2 \zeta_{\nu}^{*}\right), \\
& \eta=\zeta_{\psi}^{*},
\end{aligned}
$$

here $\zeta_{\nu}=\zeta_{\psi}-\zeta_{t}$. The exponents (5.5) - (5.8) are related by the familiar scaling laws. From the analysis given above it follows that the charged fixed point $C 2$ is the stable one and this results in the values for exponents (5.5)-(5.7) different from the values of the uncharged fixed point $\mathrm{U}$, i.e. they are not given by the ${ }^{4} \mathrm{He}$ values as it is sometimes stated (see e.g. $[2,21,5]$ ).

Recently an interesting consequence of the existence of a stable charged fixed point $\left(C_{2}\right)$ has been observed [22]. According to the renormalization of the charge (2.3) the $\beta_{f}$-function reads

$$
\beta_{f}=f\left(\varepsilon-\zeta_{A}(f, u)\right)
$$


Thus at a fixed point with $f^{*}$ nonzero the value of the gauge field $\zeta$-function is exactly given by $\zeta_{A}^{*}=\varepsilon$. That means that the penetration depth $\lambda$ and the correlation length $\xi$ are propotional and the temperature dependence follows a power law with the exponent $\nu$ [22]. At the fixed point with $f^{*}=0$ this is not the case, there we have $\zeta_{A}^{*}=0$ (each loop contribution to the $\zeta_{A}$-function contains at least one $f$-factor). Then the penetration depth behaves as $\lambda \sim \xi^{\frac{2-\varepsilon}{2}}$ and one would have two different critical length scales.

Trying to obtain the numerical values of the critical exponents on the basis of the values of fixed point $C 2$ coordinates $f^{* C, \text { Padé }}, u^{* C 2, \text { Padé }}$ given in Tables I, II in order to be self-consistent let us perform the same type of analytical continuation for the series for $\zeta$-functions, as those, which have been applyed to the $\beta$-functions (2.10), (2.11). So, introducing the auxiliary variable $t$ let us represent functions (5.5)-(5.7) in the form of resolvent series in $t$ and then we will chose the [1/1] Padé approximants for these series, which at $t=1$ will give us the analytical continuation of the series requested. Obtained in such a way expression for the critical exponent $\phi(\phi \equiv\{\nu, \gamma, \alpha)\}$ reads:

$$
\phi=a_{\phi}^{(0)} \frac{1+A_{\phi}}{1+B_{\phi}}
$$

The expressions for the coefficients $A_{\phi}, B_{\phi}$ in (5.10) read:

$$
A_{\phi}=a_{\phi}^{(1)}+B_{\phi} ; \quad B_{\phi}=-a_{\phi}^{(2)} / a_{\phi}^{(1)}
$$

and $a_{\phi}^{(i)}$ are to be determined from the resolvent series in $t$ :

$$
\phi=\left.\sum_{i \geq 0} a_{\phi}^{(i)} t^{i}\right|_{t=1}
$$

Substituting (5.2) and (5.3) into (5.5)-(5.7) and representing (5.5) - (5.7) in the form of (5.12) one finds:

$$
\begin{aligned}
& a_{\nu}^{(0)}=1 / 2, \\
& a_{\nu}^{(1)}=(n+2) / 12 u-3 / 2 f, \\
& a_{\nu}^{(2)}=\left(n^{2}-n-6\right) / 144 u^{2}+(71 n+138) / 48 f^{2}+(n+2) / 12 u f, \\
& a_{\gamma}^{(0)}=1, \\
& a_{\gamma}^{(1)}=(n+2) / 12 u, \\
& a_{\gamma}^{(2)}=\left(n^{2}-2 n-8\right) / 144 u^{2}+(5 n+1) / 4 f^{2}+5(n+2) / 24 u f, \\
& a_{\alpha}^{(0)}=1, \\
& a_{\alpha}^{(1)}=-3(n+2) / 12 u+9 / 2 f, \\
& a_{\alpha}^{(2)}=\left(-3 n^{2}+3 n+18\right) / 144 u^{2}-(71 n+138) / 16 f^{2}-(n+2) / 4 u f .
\end{aligned}
$$

Now considering the case $n=2$ and substituting coordinates of the fixed point $C 2\left(f^{* C \text {,Padé }}=.158\right)$, $u^{* C 2, \text { Padé }}=2.457$ (see Tables I, II) into (5.13)-(5.15) one obtains for the critical exponents (5.5)-(5.8) [61]:

$$
\begin{aligned}
& \nu=0.86, \quad \gamma=1.88, \\
& \alpha=-1.14, \quad \eta=-0.19 .
\end{aligned}
$$

The application of the Padé approximants for the analytical continuation of the functions may result in the appearance of poles in these functions. If the pole is located in the region of expansion parameters which is unphysical (e.g. negative coupling $u$ or $f$ ) this does not complicate the analysis. This was the case for the $\beta$ functions in the region of couplings less than the fixed point values. For the $\zeta$-functions however considering the non-asymptotic behaviour (and thus being far from the stable fixed point) one passes through a region of couplings where the Padé approximation for the $\zeta$-functions becames ambiguous resulting in the appearance of a pole. Therefore studying the crossover behaviour in the next subsection we will still keep the polynomial representa- 
tion for $\zeta$-functions instead of the Padé approximants. Then for the asymptotic values of critical exponents one gets:

$$
\begin{aligned}
& \nu=0.77, \quad \gamma=1.62, \\
& \alpha=-0.31, \quad \eta=-0.10
\end{aligned}
$$

Comparing the values (5.17) and (5.18) show a numerical difference of $15 \%$ in $\nu$ and $\gamma$ and a considerable increase of $\alpha$. However there is no qualitative change (e.g. the sign of the specific heat exponent remains the same). This should be compared with the values, given by other authors: $\nu=0.53$ and $\eta=-0.70[22]$ and $\eta=-0.38$ [23].

Effective exponents are usually defined by the logarithmic temperature derivatives of the corresponding correlation functions (see e.g. [11]). These can be found from the solutions of the renormalization group equation for the renormalized vertex functions. These effective exponents contain two contributions, one from the corresponding $\zeta$-functions now taken at the values of $u(\ell)$, $f(\ell)$ of the flow curve considered ("exponent part"), and one from the change of the corresponding scaling function ("amplitude part"). The last contributions will be neglected since we expect them to be smaller than the differences for the fixed point values of the exponents coming from the different treatments discussed before. Thus we have:

$$
\begin{aligned}
\nu & =\left(2-\zeta_{\nu}(\ell)\right)^{-1}, \\
\gamma & =\left(2-\zeta_{\nu}(\ell)\right)^{-1}\left(2-\zeta_{\psi}(\ell)\right), \\
\alpha & =\left(2-\zeta_{\nu}(\ell)\right)^{-1}\left(\varepsilon-2 \zeta_{\nu}(\ell)\right) .
\end{aligned}
$$

The flow parameter $\ell$ can be related to the relative temperature distance $T_{c}$ by the matching condition $t(\ell)=$ $\left(\xi_{0}^{-1} \ell\right)^{2}$, with $\xi_{0}$ the amplitude of the correlation length.

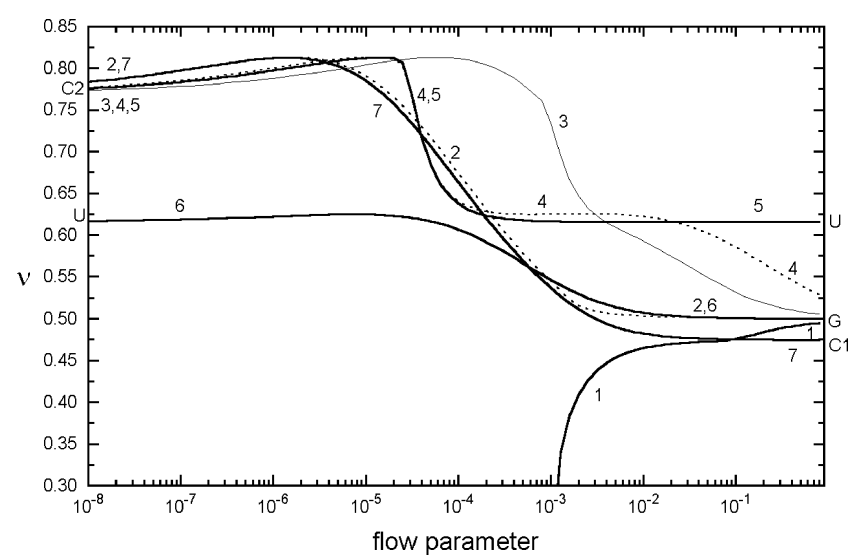

Fig. 5. Effective exponent $\nu$ for the flows shown in fig. 4 (for further description see the text).

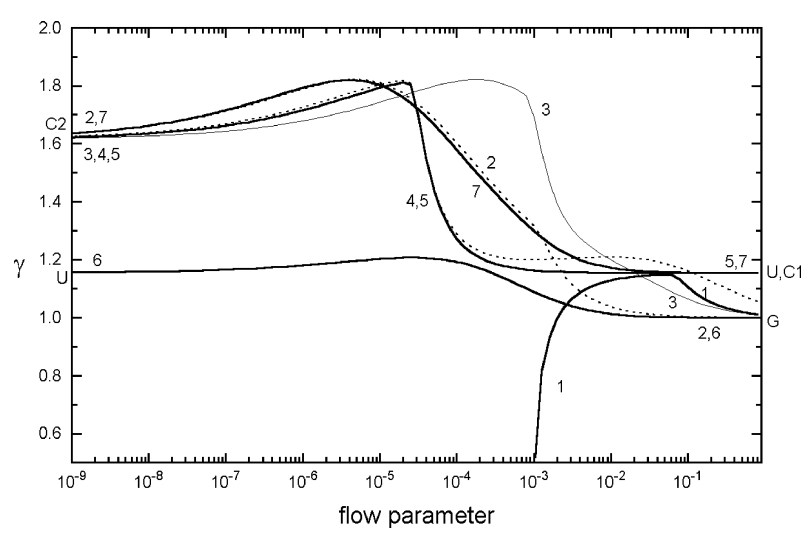

Fig. 6. Effective exponent $\gamma$ for the flows shown in fig. 4 (for further description see text).

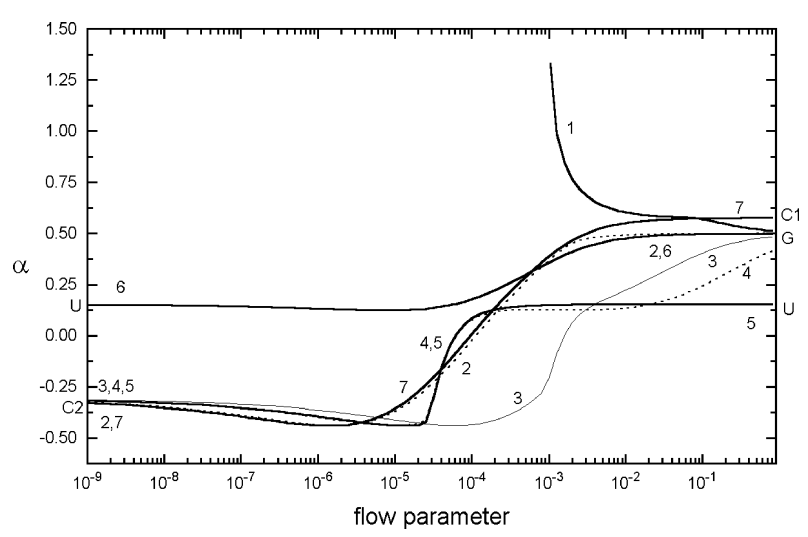

Fig. 7. Effective exponent $\alpha$ for the flows shown in fig. 4 (for further description see text).

We have computed these effective exponents, see fig. 5fig. 7, along the flow lines shown in fig. 4 by inserting [62] values of the couplings $u(\ell)$ and $f(\ell)$ into Eqs. (5.18)(5.20). For the separatrix 1 we started with initial conditions leading to a flow, which did not stick in the fixed point $\mathrm{C} 1$ but slightly missed it although the flow curve did not differ from the separatrix within the thickness of the lines shown in fig. 4 . For the curve number 4 we started somewhat further away from the Gaussian fixed point $G$ leading to the initial values of the effective exponents between their Gaussian values and their values for the uncharged fixed point $\mathrm{U}$. Note that the values of the effective exponent $\gamma$ for the uncharged fixed point $\mathrm{U}$ and the charged fixed point $\mathrm{C} 1$ are the same within the accuracy given by the scale of the figure.

\section{CONCLUSIONS}

In the present article we have re-examined expressions for the renormalization group functions of the fieldtheoretical gauge model for a superconductor obtained previously in a two-loop approximation [17]. The main point which is discussed in this context is whether the equations for $\beta$-functions possess a stable fixed point or 
not. The absence of the stable fixed point is often interpreted as a change of the order of phase transition (caused by the presence of the magnetic field fluctuations) and evidence of the fluctuation-induced first-order phase transition. However this change of the order of the phase transition (being of the second-order in the absence of the coupling to the gauge) is confirmed only by perturbation theory calculations in low orders ( [10], see [17] and the references therein as well).

In this article we applied a simple Padé analysis to the series under discussion [63].

In the case of one coupling such an approach gives a qualitatively correct picture of the phase tansition and restores the presence of a stable fixed point ( [51], see formulas (4.2), (4.6) of this article as well). The same situation happens here in the case of two couplings: at $n=2$ the "uncharged" fixed point $\mathrm{U}$ (having coordinates $f^{* U, \text { Padé }}=.158, u^{* U, \text { Padé }}=2.457$ ) appears to be stable, which leads to a new set of critical exponents. We note however that the pair correlation function critical exponent $\eta$ calculated by familiar scaling relations on the basis of sets of values (5.17) or (5.18) remains negative, which agrees with the result of [23]. Being calculated only in a two-loop approximation with the application of Padé analysis, these values for the critical exponents are to be considered as preliminary ones. The main point we claim here is that within the framework of the renormalization group analysis for the superconductor model there still exists the possibility of a second-order phase transition characterized by a set of critical exponents differing from those of ${ }^{4} \mathrm{He}$.
[1] H. Meyer-Ortmanns, Rev. Mod. Phys. 68, 473 (1996)

[2] J. Lobb, Phys. Rev. B 36, 3930 (1987).

[3] M. B. Salamon, S. E. Inderhees, J. P. Rice, B. G. Pazol, D. M. Ginsberg, N. Goldenfeld, Phys. Rev. B 38, 885 (1988).

[4] N. Overend, M. A. Howson, I. D. Lawrie, Phys. Rev. Lett. 72, 3238 (1994).

[5] I. D. Lawrie, Phys. Rev. B 50, 9456 (1994).

[6] J. A. Lipa, D. R. Swanson, J. A. Nissen, T. C. P. Chui, U. E. Israelsson, Phys. Rev. Lett. 76, 944 (1996).

[7] G. A. Baker Jr., B. G. Nickel, D. I. Meiron, Phys. Rev. B 17, 1365 (1978).

[8] J. C. Le Guillou, J. Zinn-Justin , Phys. Rev. B 21, 3976 (1980).

[9] I. A. Vakarchuk, Theor. Math. Phys. (Moscow) 36, 122 (1978).

[10] B. I. Halperin, T. C. Lubensky, S. Ma, Phys. Rev. Lett. 32, 292 (1974).

[11] J. H. Chen, T. C. Lubensky, D. R. Nelson, Phys. Rev. B 17, 4274 (1978).

[12] S. W. Lovesey, Z. Physik B Condensed Matter 40, 117 (1980)

[13] C. Dasgupta, B. I. Halperin, Phys. Rev. Lett. 47, 1556 (1981).

[14] J. Bartholomew, Phys. Rev. B 28, 5378 (1983).

[15] Let us recall that for the given Landau-Ginsburg parameter (ratio of the penetration depth to the correlation length) $k$ a superconductor with $k<1 / \sqrt{2}$ is called a type-I and the one with $k>1 / \sqrt{2}$ is called a type-II.

[16] I. D. Lawrie, C. Athorne, J. Phys. A: Math. Gen. 16, L587 (1983).

[17] S. Kolnberger, R. Folk, Phys. Rev. B 41, 4083 (1990).

[18] In fact an extensive two loop calculation where already performed earlier by M. Machacek and M. Vaughn, Nucl. Phys. B222, 83 (1983); B236, 221 (1984); B249, 70 (1985).

[19] The expression of the $\zeta$-function for the mass aggrees with C. Ford, I. Jack, D. Jones, Nucl. Phys. B387, 373 (1992)

[20] P. Arnold, L. G. Yaffe, Phys. Rev. D 49, 3003 (1994); the sign in the $\beta_{f}$-function of the two loop contribution turned out to be erroneous, otherwise the renormaliza- tion functions are in agreement with ours (private communication of the authors)

[21] M. Kiometzis, H. Kleinert, A. M. J. Schakel, Phys. Rev. Lett. 73, 1975 (1994), and Fortschr. Phys. 43, 697 (1995).

[22] I. F. Herbut, Z. Tešanović, Phys. Rev. Lett. 76, 4588 (1996).

[23] L. Radzihovsky, Europhys. Lett. 29, 227 (1995).

[24] A. J. Bray, Phys. Rev. Lett. 32, 1413 (1974).

[25] P. G. de Gennes, Solid State Commun. 10, 753 (1972).

[26] T. C. Lubensky, J.-H. Chen, Phys. Rev. B 17, 366 (1978).

[27] G. B. Kasting, K. J. Lushington, C. W. Garland, Phys. Rev. B 22, 321 (1980).

[28] M. A. Anisimov, P. E. Cladis, E. E. Gorodetskii, D. A. Huse, V. E. Podneks, V. G. Taratuta, W. van Saarloos, V. P. Voronov, Phys. Rev. A 41, 6749 (1990).

[29] see e.g. X. Wen, Y. Wu, Phys. Rev. Lett. 70, 1501 (1993) and L. Pryadko, S. Zhang, Phys. Rev. Lett. 73, 3282 (1994).

[30] G.H. Hardy, Divergent Series, (Oxford University, Oxford) 1948 .

[31] J. C. Le Guillou, E. Brézin, J. Zinn-Justin, Phys. Rev. D, 15, 1544 (1977).

[32] L. N. Lipatov, Sov. Phys. JETP. 45, 216 (1977).

[33] E. Brezin, G. Parisi, J. Stat. Phys. 19, 269 (1978).

[34] R. Folk, Yu. Holovatch, J. Phys. A: Math. Gen. 29, 3409 (1996).

[35] B. Bergerhoff, F. Freire, D. F. Litim, S. Lola, C. Wetterich, Phys. Rev. B 53, 5734 (1996)

[36] G. t'Hooft, M. Veltman, Nucl. Phys. B 44, 189 (1972).

[37] R. Schloms, V. Dohm, Nucl. Phys. B 328, 639 (1989).

[38] R. Schloms, V. Dohm, Phys. Rev. B 42, 6142 (1990).

[39] G. Grinstein, A. Luther, Phys. Rev. B 13, 1329 (1976).

[40] R. Schloms, V. Dohm, Europhys. Lett. 3, 413 (1987).

[41] Only for the case when one of the couplings is equal to zero one obtains a series which is proved to be the asymptotic one.

[42] G. Jug, Phys. Rev. B 27, 609 (1983).

[43] I. O. Mayer, A. I. Sokolov, B. N. Shalaev, Ferroelectrics 95, 93 (1989).

[44] I. O. Mayer, J. Phys. A 22, 2815 (1989).

[45] H. K. Janssen, K. Oerding, E. Sengespeick, J. Phys. A: 
Math. Gen. 28, 6073 (1995).

[46] P. W. Mitchell, R. A. Cowley, H. Yoshizawa, P. Böni, Y. J. Uemura, R. J. Birgeneau, Phys. Rev. B 34, 4719 (1986).

[47] T. R. Thurston, C. J. Peters, R. J. Birgeneau, P. M. Horn, Phys. Rev. B 37, 9559 (1988).

[48] J.-S. Wang, M. Wöhlert, H. Mühlenbein, D. Chowdhury, Physica A 166, 173 (1990).

[49] J.-S. Wang, W. Selke, Vl. S. Dotsenko, V. B. Andreichenko, Europhys. Lett. 11, 301 (1994); A. L. Talapov, L. N. Shchur, Europhys. Lett. 27, 193 (1994).

[50] T. Holey, M. Fähnle, Phys. Rev. B 41, 11709 (1990).

[51] G. Parisi, in Proceedings of the 1973 Cargrése Summer School unpublished. G. Parisi, J. Stat. Phys. 23, 49 (1980).

[52] Yu. Holovatch, Preprint, C.E.Saclay, Service de Physique Theorique; SPhT/92-123); Yu. Holovatch, Int. J. Mod. Phys. A 8, 5329 (1993).

[53] The last possibility was chosen by Parisi [51] in order to restore the presence of stable solution for the fixed point in two-loop approximation.

[54] The series in (4.1) appears to be the alternating one and this scheme can be applied without any difficulties.
[55] M. Abramowitz, A. I. Stegun, (editors), Handbook of Mathematical Functions with Formulas, Graphs and Mathematical Tables (National Bureau of Standards, 1964).

[56] In this case the principal value of the integral (3.4) could be taken, but generally speaking it is preferable to avoid such situations (see [7] as well).

[57] J. S. R. Chisholm, Math. Comp. 27, 841 (1973).

[58] G. A. Baker Jr., P. Graves-Morris, Padé approximants (Addison-Wesley Publ. Co., Reading, Mass.) (1981).

[59] P. J. S. Watson, J. Phys. A 7, L167 (1974).

[60] We take $\ell_{0}=1$.

[61] The value of $\eta$ has been found by the scaling law: $\eta=2-\gamma / \nu$.

[62] In fact we have solved the flow equations (4.20), (4.21) starting near the unstable fixed points, for the initial value of the flow parameter we took $\ell=1$. Using different initial values $(u(1)$ and $f(1)$ on the seperatrix) would amount to rescale the flow parameter.

[63] Because they are double series in two coupling constants we made use of representing them in the form of resolvent series $[59,58]$ which enabled us then to pass to Padé analysis.

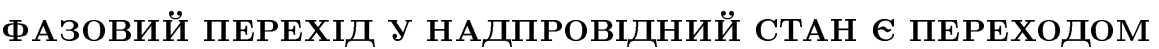 ПЕРШОГО ЧИ ДРУГОГО РОДУ?
}

\author{
Р. Фольк ${ }^{\dagger}$, Ю. Головач ${ }^{\dagger \dagger}$ \\ ${ }^{\dagger}$ Інститут теоретичної фізики, університет Йогана Кеплера, А-4040 Ліни, Австрія \\ ${ }^{\dagger \dagger}$ Інститут фізики конденсованих систем НАН Украӥни \\ Украӥна, 290011, Лъвів, вул. Свенцічъкого, 1
}

\begin{abstract}
Досліджено фазовий перехід у надпровідний стан з урахуванням флюктуацій параметра впорядкування і векторного магнетного поля та обговорено питання про рід фазового переходу у такій моделі. Ми використовуємо теоретико-польовий ренормгруповий підхід і розглядяємо теоретико-польову калібрувальну модель надпровідника, узагальнену на випадок $n / 2$ компонентного комплексного параметра впорядкування. Попередні ренормгрупові обчислення з безпосереднім $\varepsilon$-розкладом свідчили про те, що у такій моделі відбувається фазовий перехід першого роду. Ми ще раз проаналізували вирази для ренормгрупових функцій у двопетлевому наближенні в тривимірному просторі. Особливу увагу звернуто на те, що відповідні ряди можуть бути асимптотичними і мати нульовий радіус збіжності. Ми обговорюємо можливі способи аналітичного продовження отриманих рядів. На підставі порівняння результатів, отриманих за допомогою "безпосередніх" обчислень, Паде-аналізу та техніки Паде-Борелівського пересумовування зроблено таке припущення: у моделі є можливість фазового переходу другого роду з критичними показниками, що відрізняються від критичних показників надплинної рідини. Це припущення узгоджується з висновками, зробленими недавно за допомогою непертурбативних підходів.
\end{abstract}

